\title{
Integrin Dimerization and Ligand Organization: Key Components in Integrin Clustering for Cell Adhesion
}

\author{
CHRISTOPHER J. BRINKERHOFF, M.S., and JENNIFER J. LINDERMAN, Ph.D.
}

\begin{abstract}
Cell adhesion requires both integrin occupancy and integrin clustering. In this work, we investigate a mechanism based on organizing ligand into islands and integrin dimerization for the initiation of integrin clustering. To study integrin clustering and integrin occupancy we develop a two-dimensional Monte Carlo lattice description of the cell-substrate interface to simulate the diffusion and reaction of integrins. We demonstrate that integrin dimerization can drive integrins into clusters of sizes greater than two. Ligand organization or integrin dimerization alone is unable to increase the number of bound integrins, but when both are present they cooperate to increase both binding and clustering of integrins. In addition, when integrin dimerization and ligand organization are both present large integrin clusters, which may act as nucleation sites for the formation of adhesion complexes, are observed. These results describe a potential mechanism for the clustering of integrin receptors and avidity modulation in cellular adhesion and have implications for the designs of surfaces to control cell responses to external ligands and to manipulate cell adhesion for tissueengineering applications.
\end{abstract}

\section{INTRODUCTION}

A DHESION RECEPTORS govern cell adhesion to physical structures in the environment, for example, other cells and artificial surfaces. Adhesion receptors termed integrins are the primary mechanism by which cells attach to the extracellular matrix (ECM). Integrins are composed of two noncovalently associated subunits ( $\alpha$ and $\beta$ ) that form a binding pocket for specific sequences or domains in ECM molecules; the most well known is the tripeptide RGD domain found in fibrinogen and other ECM molecules. After the binding of an integrin to its ligand, an aggregate of integrins and other intracellular proteins forms an adhesion complex, connecting the integrins to the actin cytoskeleton and activating many signaling pathways involved in adhesion. ${ }^{1}$ Integrins restrained from forming clusters via mutations in the $\alpha$ - or $\beta$-chain cytoplasmic tail have impaired cell adhesion., ${ }^{2,3}$
The importance of clustering of integrins for ligand binding in particular was demonstrated by nonphysiological techniques such as chemical cross-linking agents or multivalent antibodies. ${ }^{4}$ In this article, we investigate integrin dimerization, a naturally occurring property of integrins that may drive the initial clustering of integrins, and the impact of this clustering on ligand binding.

One physiological mechanism that produces integrin clustering is binding to multivalent ligands. Many of the ECM proteins that integrins bind are multimeric, for example, tenacin- $\mathrm{C}^{5}$ and polymerized networks of fibronectin molecules. ${ }^{6}$ By tethering many RGD peptides to a polymer or by depositing RGDs on a surface, artificial multivalent ligands have been created. ${ }^{7-9}$ These experiments revealed that RGD peptides presented singly (one peptide per polymer molecule) are poor substrates for adhesion, whereas the same concentration of peptides induces greater adhesion when presented as a multiva-

Department of Chemical Engineering, University of Michigan, Ann Arbor, Michigan. 
lent molecule (many peptides in the same polymer molecule). Clearly, the organization of ligand has a role in integrin clustering.

A second mechanism for causing clusters (two or more integrins) is integrin dimerization. Several groups have observed dimers and oligomers of integrins formed by simple protein-protein interactions independent of ligand binding. The $\beta_{1}$ subunit self-associates to form clusters on the cell surface ${ }^{3}$ and the $\beta_{2}$-integrin subunit clusters on the surface of leukocytes. ${ }^{10}$ The $\alpha_{\text {IIb }}$ and the $\beta_{3}$ subunits both can form clusters via a weak interaction between subunits to create clusters of two or three integrins. ${ }^{11,12}$ In addition, blocking integrin dimerization by overexpression of the integrin $\beta_{1}$-subunit cytoplasmic tail decreases integrin clustering and reduces cell adhesion. ${ }^{3}$ However, the role that integrin dimerization has in integrin function has been difficult to determine.

Integrin clustering is a necessary step in the process of assembling adhesion complexes. The larger structures are visible by light microscopy, but smaller nascent integrin clusters are not. ${ }^{13}$ Yet these initial clusters of only a few integrins have an important role in initiating adhesion and may recruit functionally important signaling molecules that do not partition to the larger adhesion complexes. ${ }^{14,15}$ New experimental methods may allow visualization of the interactions between integrins during the initial stages of adhesion ${ }^{16}$ and direct quantification of these initial clusters has been reported. ${ }^{17}$ In this article, we explore the use of computer models for observing the organization of integrins and ligands in the initiating events of adhesion complex formation.

We propose that the dimerization of integrins creates large (more than two integrins) clusters and initiates the assembly of even larger integrin complexes. An analogous, weak protein-protein dimerization among $\mathrm{G}$ protein-coupled receptors has been shown to create receptor clusters. ${ }^{18}$ Precisely because integrin dimerization is weak, it may cause significant clustering of integrins. This weak interaction allows the switching of bonds between neighboring integrins, or "partner switching," to occur quickly relative to the diffusion rate for integrins. This allows multiple neighbors to effectively share a single bond and creates a cluster of integrins larger than a dimer pair, as shown schematically in Fig. 1. For such clustering of integrins to occur, the rate of dimerization must be fast relative to the rate of diffusion. In other words, the reaction is diffusion limited, as we would expect for the interaction of two molecules in the cell membrane. ${ }^{19}$ Therefore, we developed a computer simulation of the cell-substrate interface that models integrin diffusion in addition to integrin dimerization and binding to ligand.

We use our simulations to test the hypothesis that specific arrangements of ligand, together with integrin dimerization, can enhance both the binding and cluster-

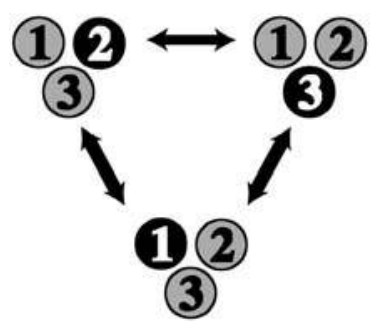

FIG. 1. Dimerization of integrins can lead to the formation of oligomers via partner switching. Integrins are represented as circles. The dimerized pairs of integrins are gray and monomers are black.

ing of integrins. These results have implications for understanding the initiation of integrin clustering and integrin binding to ligand, both important in cell adhesion. We also predict the most useful arrangement of ligand to enhance cellular adhesion for use in the design of biomimetic surfaces.

\section{MATERIALS AND METHODS}

To examine the roles of ligand organization and integrin dimerization in ligand binding and clustering, we developed a Monte Carlo simulation of integrin dimerization and integrin-ligand complex formation at the cell-substrate interface. The cell membrane was assumed to be flat against the substrate with a separation of 30 $\mathrm{nm}$, approximately the distance over which integrins can interact with ligand. ${ }^{19}$ A two-dimensional lattice was used to represent the cell membrane and cell surface molecules. Simulations were run on a 1000 by 1000 triangular lattice with periodic boundary conditions and a lattice spacing $(l)$ of $1.5 \mathrm{~nm}$. Using this lattice spacing allowed us to approximate diffusion on a continuous surface. This simulated a total area of $2.25 \mu \mathrm{m}^{2}$, one order of magnitude larger than the area of a fully formed adhesion complex (area, $\sim 0.1 \mu \mathrm{m}^{2}$ ). ${ }^{20}$ Larger lattices simulating up to $20 \mu \mathrm{m}^{2}$ produced no significant differences in results.

The lattice contained integrin receptors simulated as hexagons with a diameter of eight lattice spacings. This corresponds to an area of approximately $100 \mathrm{~nm}^{2}$, roughly the size of an integrin molecule. ${ }^{21}$ The number of integrins on the surface was set at 1000 integrins $/ \mu \mathrm{m}^{2}$. This surface concentration is an approximate value taken from the typical expression levels of many different cell and integrin types ${ }^{22-27}$ and assuming that the integrins are evenly distributed. The effect of the number of integrins was evaluated and while changing the number causes quantitative differences in the results, the qualitative trends remained consistent. Therefore, these findings reassure us that our choice of 
integrin concentration is not critical to the characteristic behavior of the system.

\section{Ligand organization}

The substrate containing ligand molecules in a particular organization was assumed to lie parallel to the cell membrane. The locations on the cell membrane lattice opposite a ligand molecule on the substrate were marked as ligand-binding sites before the beginning of the simulation. Ligand-binding sites on the cell membrane were simulated as hexagons with a diameter of one lattice space, which corresponds to an area of approximately $2.25 \mathrm{~nm}^{2}$, roughly the size of a small peptide, that is, the RGD-binding site. ${ }^{29}$ The number of ligands on the substrate was varied an order of magnitude, from 180 to 1800 ligands $/ \mu \mathrm{m}^{2}$. This broad range encompasses the range used in experiments with controlled ligand organization. ${ }^{9,28}$

Ligand was placed on the substrate in one of two ways: randomly or grouped in islands (Fig. 2). Ligands remained immobile for the duration of the simulation. For ligand grouped in islands each island was randomly placed on the substrate without overlapping another island. Ligand islands mimic a multivalent ligand by creating regions of high ligand concentration without using a high overall ligand concentration. For most simulations, the size of the ligand islands was held constant at nine ligands per island and the spacing between ligands in an island was $15 \mathrm{~nm}$. At the concentrations of ligand used (180-1800 ligands $/ \mu \mathrm{m}^{2}$ ), the average spacing between islands $(62-220 \mathrm{~nm})$ was sufficiently large to separate the islands. At higher ligand concentrations (>6000 ligands $/ \mu \mathrm{m}^{2}$ ) the ligands would no longer be in islands, because the distance between ligands of two different islands $(<20 \mathrm{~nm})$ would not be significantly different from the distance between ligands within the same island (15 $\mathrm{nm})$. The 15-nm spacing between ligands in an island was used because it minimized steric limitations of ligand availability, but was not so large as to negate the bene- fit of a high local ligand concentration. This is similar to the spacing reported by Koo et al. ${ }^{9}$

\section{Simulation algorithm}

Simulations used an algorithm similar to previous models. ${ }^{18}$ Briefly, integrins were chosen at random to attempt one of five randomly chosen actions. The five possible actions were as follows: dimerization with a neighbor, dissociation from a dimerized pair, binding to ligand, dissociation from ligand, or diffusion in the plane of the membrane.

If the chosen action was to dimerize with a neighbor, the integrin was first tested to determine whether it was a monomer. Then a random neighboring integrin within the interaction radius of four lattice spacings $(6 \mathrm{~nm}$, the maximum distance at which two integrins can dimerize) was tested to determine whether it was also a monomer. If so, dimerization was allowed with probability $P_{\text {dimer }}$. If the chosen action was to dissociate from a dimerized pair, the integrin was checked to determine whether it was a dimer and dissociation was allowed with a probability $P_{\text {mono }}$ (calculation of probabilities is detailed below).

If the chosen action was to bind ligand, the integrin was first tested to determine that it was not bound to ligand already. Then the area within the binding radius of two lattice spacings (a hexagonal area of $27 \mathrm{~nm}^{2}$ ) was searched for a free ligand-binding site. If a ligand-binding site was found, this integrin and ligand then became a bound complex with probability $P_{\text {bind }}$. Integrin-ligand complexes were allowed to disassociate with probability $P_{\text {unbind. }}$

For a diffusion event, integrins moved a single lattice space in a random direction according to the rules outlined by Woolf and Linderman ${ }^{18}$ and briefly explained below. If the new location was occupied, then the move was rejected and not repeated. If the integrin was bound to ligand it was not able to move. If the integrin was part of a dimer, then additional constraints were placed on its

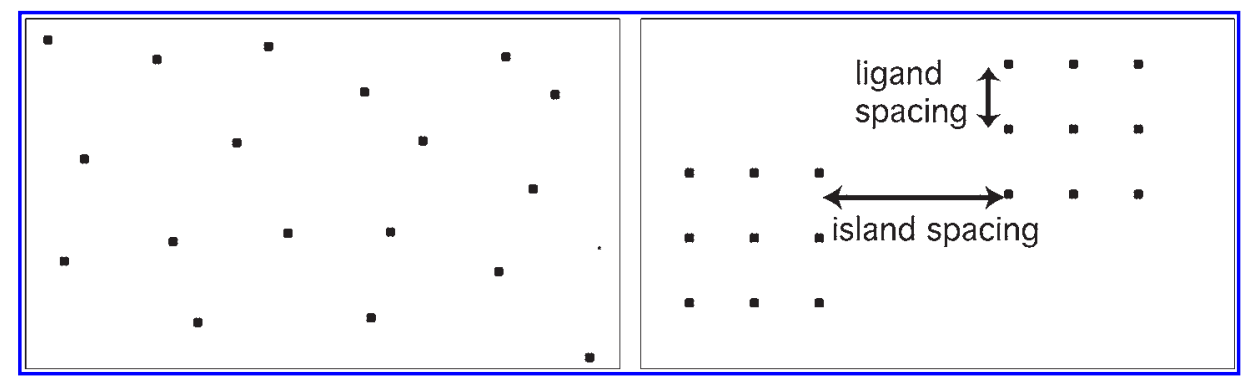

FIG. 2. Examples of random ligand placement (left) and organization into ligand islands (right). Individual ligand-binding regions, that is, an RGD tripeptide, are represented with black dots. For the ligand islands, the distance between one ligand and its nearest neighbor is termed the ligand spacing and was typically $15 \mathrm{~nm}$, but for random ligand organization the average distance between a ligand and its nearest neighbor is much greater. 
movement. A single integrin within a dimer was allowed to move around its partner or move closer toward its partner without causing the integrins to overlap. If an integrin attempted to overlap or move away from its partner, then an attempt was made to move both integrins. As a result of these rules, integrins move with approximately the same diffusion coefficient independent of dimer state. This property is consistent with theoretical findings that show the diffusion of integrins is not effected by oligomerization. ${ }^{30}$

To determine the effects of ligand organization and integrin dimerization on integrin-mediated adhesion, we measured three parameters, the number of integrin-ligand complexes, the average integrin cluster size, and the cluster size distribution, all indicators of the initial events in cellular adhesion. The average cluster size was measured by counting the total number of integrins that were within the interaction radius of at least one member of the same cluster. Clusters generally include a mixture of both monomers and dimers (Fig. 3). However, a cluster could exist without any interaction between neighbors if integrins happened to be near each other. To determine the cluster size distribution the fraction of integrins in each size cluster was measured.

Before data were collected, all simulations were allowed to preequilibrate. Simulations reached equilibrium in what corresponds to a real time of milliseconds. After equilibration, at least 100 distinct measurements were collected. The total real time simulated was on the order of $10 \mathrm{~ms}$. Simulations were written in $\mathrm{C}++$ and run on a cluster of Apple G4 machines. Source code is available from the authors on request.

\section{Parameter estimation}

For discrete time models, the dimerization and monomerization reactions can be modeled as Poisson processes and the probabilities of reaction can be derived from the bulk reaction rates, using a Poisson distribution. With sufficiently small time steps $(\Delta t)$, the probabilities of reaction are nearly proportional to the intrinsic dimer-

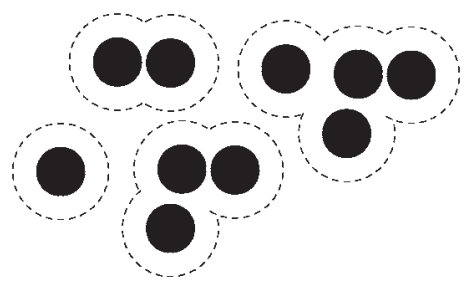

FIG. 3. Examples of integrin clusters of sizes 1, 2, 3, and 4. Integrins are represented as black circles and clusters are surrounded by a dotted line. Although dimerization may cause integrin clustering, clusters are measured solely by the distance between integrins and not by the dimerization state. ization and monomerization reaction rate constants, $k_{\text {dimer }}$ and $k_{\text {mono }}$, respectively, according to Rowley ${ }^{31}$ :

$$
\begin{aligned}
& P_{\text {dimer }}=1-\exp \left(-k_{\text {dimer }} \Delta t\right) \approx k_{\text {dimer }} \Delta t \\
& P_{\text {mono }}=1-\exp \left(-k_{\text {mono }} \Delta t\right) \approx k_{\text {mono }} \Delta t
\end{aligned}
$$

The intrinsic rate constants $k_{\text {dimer }}$ and $k_{\text {mono }}$ describing dimerization and monomerization on the two-dimensional membrane surface were estimated from literature data as follows. Li et al. ${ }^{11}$ measured the equilibrium dissociation constant for the dimerization of two integrins in solution, $K_{\mathrm{D} \text {,dimer, }}^{3 \mathrm{~d}}$ to be $0.0001-0.01 \mathrm{M}$. The equivalent value of the equilibrium constant in two dimensions can be found from

$$
K_{\mathrm{D}, \text { dimer }}^{2 \mathrm{~d}}=\frac{K_{\mathrm{D}, \mathrm{dimer}}^{3 \mathrm{~d}}}{(4 / 3) R_{\mathrm{enc}}}
$$

where $K_{\mathrm{D} \text {,dimer }}^{2 \mathrm{~d}}$ is the equilibrium dissociation constant in two dimensions and is equal to the ratio of the bulk reverse reaction rate constant to the bulk forward reaction rate constant $\left(k_{\text {off }} / k_{\text {on }}\right) . R_{\text {enc }}$ is the encounter radius between two integrin monomers, estimated as 2.5 nm. ${ }^{32}$ The factor $4 / 3$ accounts for differences in the geometry. ${ }^{33}$

For monomerization, the intrinsic reaction rate constant $k_{\text {mono }}$ is equivalent to the bulk reaction rate constant $k_{\text {off. }}$. However, for dimerization the bulk reaction rate constant $k_{\text {on }}$ must be split into two parts, the intrinsic reaction rate constant $k_{\text {dimer }}$ and the transport rate constant $k_{+}$. The transport rate constant, $k_{+}$, is dependent only on the geometry of the reaction, so it can be estimated by Shoup and $\mathrm{Szabo}^{34}$ :

$$
k_{+}=\frac{2 \pi D_{\mathrm{t}}}{\ln \left(b / R_{\mathrm{enc}}\right)}
$$

where $D_{\mathrm{t}}$ is the translational diffusion coefficient of integrins in the cell membrane, estimated as $10^{-11}-10^{-10}$ $\mathrm{cm}^{2} / \mathrm{s},{ }^{2,17,35} b$ is one-half the mean distance between integrins, calculated as $18 \mathrm{~nm}$ for this integrin concentration, and $R_{\text {enc }}$ is the encounter radius between two integrin monomers, estimated as $2.5 \mathrm{~nm}$.

By assuming that $k_{+}<<k_{\text {dimer }}$ (implying the reaction is diffusion limited and the bulk reaction rate constant $k_{\text {on }}$ is dominated by $k_{+}$, as would be expected for an interaction between two molecules in the cell membrane $^{19}$ ) the intrinsic reaction rate constant describing monomerization can be determined from $k_{+}$and $K_{\mathrm{D} \text {,dimer }}^{2 \mathrm{~d}}$ by

$$
k_{\text {mono }}=K_{\mathrm{D}, \text { dimer }}^{2 \mathrm{~d}} \cdot k_{+}
$$

We find that $k_{\text {mono }}$ is on the order of $10^{1}-10^{3} \mathrm{~s}^{-1}$. To determine $k_{\text {dimer }}$, we set the equation for the probability of reaction $[\mathrm{Eq} .(2)]$ equal to the fraction of reaction at- 
tempts that are successful, or the "capture probability" $\gamma,{ }^{19}$ and solve for $k_{\text {dimer }}$ by

$$
k_{\text {dimer }} \Delta t=\gamma=\frac{k_{\text {dimer }}}{k_{\text {dimer }}+k_{\text {mono }}}
$$

where $\Delta t$ is the size of a time step in the simulation, set at $10^{-6} \mathrm{~s}$. We find that $k_{\text {dimer }}$ is approximately $10^{5} \mathrm{~s}^{-1}$, similar to that estimated for $\mathrm{G}$ protein-coupled receptors. ${ }^{18}$

The probabilities of ligand binding and dissociation events were estimated from the bulk reaction rate constants $k_{\text {bind }}$ and $k_{\text {unbind }}$ reported in the literature. The value of $k_{\text {unbind }}$ has been measured to be in the range 0.2-22 $\mathrm{s}^{-1}$ for the dissociation of $\alpha_{\mathrm{IIb}} \beta_{3}$ integrins from an RGD molecule. ${ }^{36,37}$ For a ligand binding event we again need to account for the two-dimensional geometry inherent in binding to an adhesion molecule by using Eq. (3). For an integrin binding to an RGD peptide $K_{\mathrm{D} \text {,dimer }}^{3 \mathrm{~d}}$ is $\sim 10^{-6}-10^{-7} \mathrm{M}^{38}$ and the encounter radius, $R_{\mathrm{enc}}$, is $\sim 0.1$ $\mathrm{nm} \cdot{ }^{36}$ Using

$$
k_{\text {bind }}=\frac{k_{\text {unbind }}}{K_{\mathrm{D} \text {,dimer }}^{2 \mathrm{~d}}}
$$

we then calculate that $k_{\text {bind }}$ is $\sim 10^{-8}-10^{-6} \mathrm{~cm}^{2} / \mathrm{s}$.

The probability of a diffusion event, $P_{\text {move }}$, was calculated using the translational diffusion coefficient $D_{\mathrm{t}}$ of proteins in a lipid bilayer. For a single particle exhibiting Brownian diffusion on a triangular lattice, the probability of a particle moving at least one lattice spacing, $l$, in one iteration time step, $\Delta t$, can be approximated with

$$
P_{\text {move }}=1-\exp \left(\frac{-6 \Delta t D_{\mathrm{t}}}{l^{2}}\right) \approx \frac{6 \Delta t D_{\mathrm{t}}}{l^{2}}
$$

The value of $D_{\mathrm{t}}$ is in the range of $10^{-11}-10^{-10} \mathrm{~cm}^{2} / \mathrm{s}$, corresponding to a range of characteristic rates for moving, $k_{\text {move }}$, of $10^{4}-10^{5} / \mathrm{s}$.

\section{RESULTS}

We used our simulations to examine the roles that integrin dimerization and ligand organization (both alone and together) play in determining integrin binding and clustering. We focus on three key quantities: the total amount of ligand bound, the average integrin cluster size, and the integrin cluster size distribution.

\section{Integrin dimerization without ligand binding: integrin dimerization increases average integrin cluster size}

We first simulated integrin dimerization in the absence of ligand and observed the effect of integrin dimerization on the average integrin cluster size and cluster size distribution. When integrins are unable to dimerize $\left(k_{\text {dimer }}=\right.$ $\left.0, K_{\mathrm{D}, \text { dimer }}^{3 \mathrm{~d}}=\infty\right)$, the average cluster size is 1.5 . The av- erage cluster size is greater than one (the minimum possible value) because some integrins by chance are found close enough to each other to be counted as a cluster (see Fig. 3). When integrins dimerize irreversibly $\left(k_{\text {mono }}=0\right.$, $K_{\mathrm{D} \text {,dimer }}^{3 \mathrm{~d}}=0$ ) the average cluster size is 2.6 (Fig. 4). This tight dimerization does not allow the integrins to switch partners (Fig. 1) and therefore large oligomers do not form. The average cluster size is greater than two because some dimer pairs by chance are found sufficiently close to another dimer pair to be counted as part of the same cluster.

We then ran simulations using a physiologically relevant value for dimerization $\left(K_{\mathrm{D} \text {,dimer }}^{3 \mathrm{~d}}=0.01 \mathrm{M}^{11}\right)$. At this intermediate value of $K_{\mathrm{D} \text {,dimer }}^{3 \mathrm{~d}}$, partner switching is significant, in agreement with the results of previous simulations for $\mathrm{G}$ protein-coupled receptors. ${ }^{18}$ The average cluster size is increased to 3.1 (Fig. 4). Approximately $15 \%$ of the integrins are found in clusters of greater than eight integrins while for $K_{\mathrm{D}, \text { dimer }}^{3 \mathrm{~d}}=0$ less than $4 \%$ of the integrins are found in such large clusters.

\section{Ligand organization without integrin} dimerization: ligand organization increases average integrin cluster size but not the number of bound ligands

To assess the impact of ligand organization, we next simulated integrin binding (in the absence of dimerization) to ligand arranged randomly or in ligand islands. Ligand arranged in islands mimicks a multivalent ligand by creating regions of high local ligand concentration

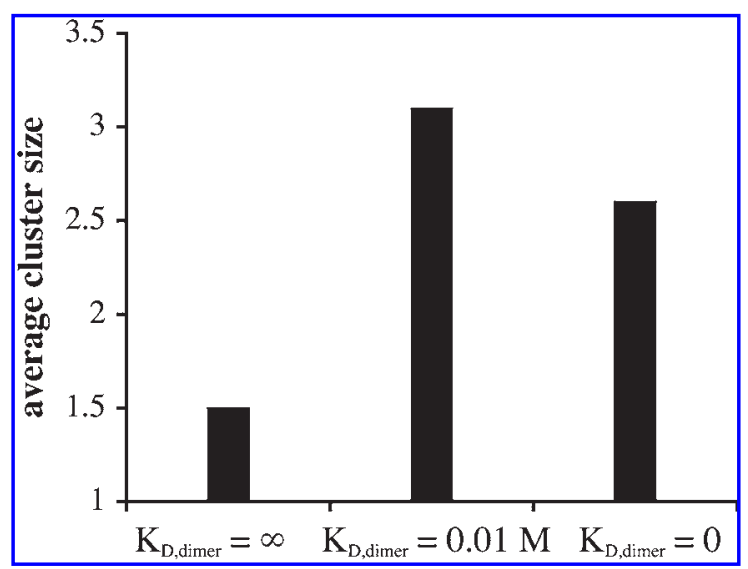

FIG. 4. Clustering of integrins as a result of dimerization. No ligand is present. The average cluster size is plotted for three values of $K_{\mathrm{D} \text {,dimer }}^{3 \mathrm{~d}}$. At intermediate values of $K_{\mathrm{D} \text {,dimer }}^{3 \mathrm{~d}}$ partner switching can occur and oligomers (clusters with more than two integrins) are observed. The fraction of integrins in clusters greater than size 8 is less than $0.1 \%$ for $K_{\mathrm{D} \text {,dimer }}^{3 \mathrm{~d}}=\infty$, less than $4 \%$ for $K_{\mathrm{D} \text {,dimer }}^{3 \mathrm{~d}}=0$, and $15 \%$ for $K_{\mathrm{D} \text {,dimer }}^{3 \mathrm{~d}}=0.01 \mathrm{M}$. Parameters: $k_{\text {dimer }}=0$ or $10^{5} \mathrm{~s}^{-1}, k_{\text {mono }}=0$ or $10^{3} \mathrm{~s}^{-1}, k_{\text {move }}=10^{4}$ $\mathrm{s}^{-1},[\mathrm{~L}]=0$. 
while the average concentration is set equal to that for random placement of ligand.

When ligand is placed randomly, the concentration of ligand has no effect on the cluster size. The cluster size is greater than one because some integrins are by chance alone close enough to each other to count as a cluster [as can be calculated with Eq. (8)]. When ligand islands are present, the average cluster size is increased up to 3-fold as compared with randomly placed ligand (Fig. 5a), and the cluster size is a strong function of the ligand density. At low ligand concentrations, many of the integrins are unable to find free ligand because little is available. These unbound integrins remain randomly distributed and keep the average cluster size relatively small. At intermediate ligand concentrations, the numbers of integrins and ligand molecules are similar. The integrin and the ligand must be in the same location to bind and therefore the presence of ligand islands causes the integrins to adopt the ligand organization. The increase in average cluster size for the organized ligand as compared with the randomly placed ligand indicates this increase in integrin organization. Finally, at high ligand concentrations, the ligand is in great excess and so integrins are able bind ligand and remain randomly spread on the surface.
The number of bound integrins increases with increasing ligand concentration (Fig. 5b). The analytical result from Lauffenburger and Linderman ${ }^{19}$ for the equilibrium number of bound integrins is recovered when the size of an individual integrin in our simulation is reduced to approximate the assumption of infinitely small particles inherent in the analytical solution (data not shown).

\section{Integrin dimerization with binding to randomly placed ligand: dimerization reduces binding}

When integrins are able to dimerize as well as bind to randomly placed ligand, the number of bound ligands is reduced and the average cluster size is increased as compared with nondimerizing integrins (Fig. 6). Image correlation microscopy has been used to observe the in vivo organization of integrins. Clusters with an average size of four were observed, similar to the results of our simulations with dimerization and binding to randomly arranged ligand. ${ }^{17}$ Integrins are less able to find an available ligand because the ligand is randomly spread on the surface, but the integrins are clustered because of their dimerization. An example of this competition between integrin-integrin and integrin-ligand interactions is dem-
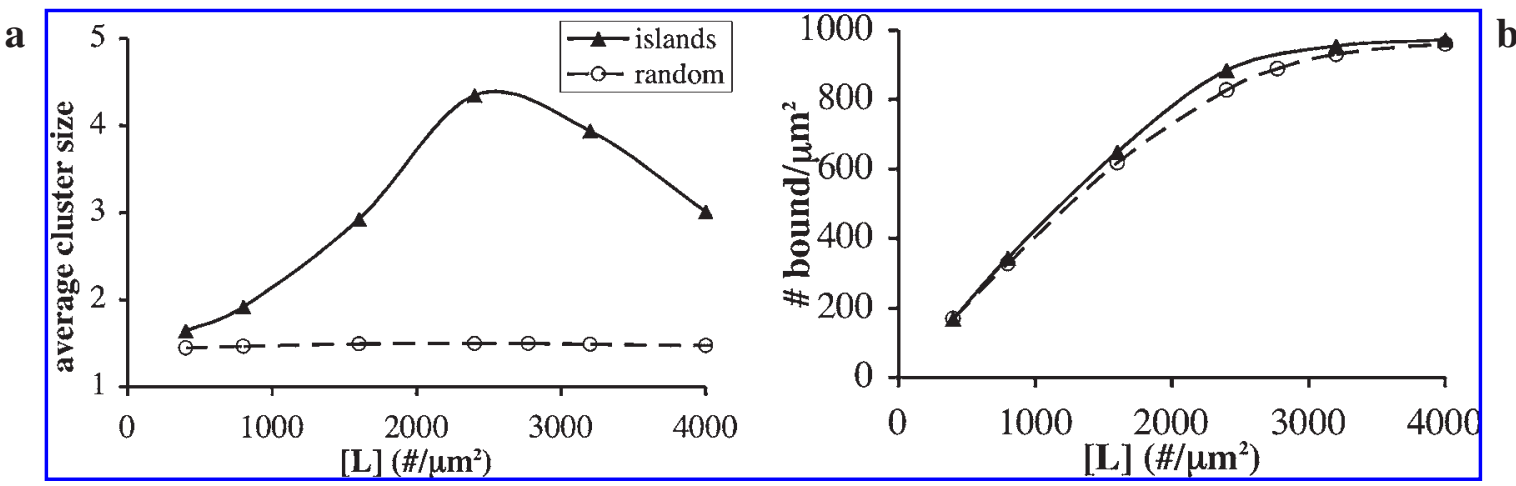

FIG. 5. Clustering and binding of nondimerizing integrins as a result of ligand organization. (a) The average cluster size and (b) the number of bound integrins versus ligand density [L] are plotted for randomly arranged ligands and ligand islands (nine ligands per island; $15 \mathrm{~nm}$ between ligands in the same island). The organization of ligand has a small effect on the number of bound integrins because randomly placed ligands are occasionally placed too close together to be simultaneously bound to an integrin. When only available ligands are considered the number of bound integrins is the same for ligands randomly arranged and ligand islands.* We also measured the distribution of integrin cluster sizes. When no ligand is present, $15 \%$ of the integrins are in clusters greater than size 8 . For a total ligand concentration of $2400 / \mu \mathrm{m}^{2}$ randomly arranged $28 \%$ of the integrins are in clusters greater than size 8 and arranged in islands $82 \%$ of the integrins are in clusters greater than size 8 . Parameters: $k_{\text {dimer }}=0 \mathrm{~s}^{-1}$, $k_{\text {mono }}=0 \mathrm{~s}^{-1}, k_{\text {bind }}=10^{-7} \mathrm{~cm}^{2} / \mathrm{s}, k_{\text {unbind }}=1 \mathrm{~s}^{-1}, k_{\text {move }}=10^{5} \mathrm{~s}^{-1}$.

*The probability, $P$, of placing a ligand too close to another ligand to allow integrin binding is equal to the area excluded by already placed ligands divided by the total area, or

$$
P=\frac{N \cdot A}{L^{2}}
$$

where $N$ is the number of ligands already placed, $A$ is the area of one integrin $\left(100 \mathrm{~nm}^{2}\right)$, and $L^{2}$ is the total area of the simulation grid $\left(2.25 \mu \mathrm{m}^{2}\right)$. The number of ligand pairs that have one inaccessible ligand is $P$ times the number of ligands. One-half of these inaccessible ligand pairs (one of the pair can be bound, not both) is subtracted from the total number of ligands to obtain the number of available ligands. The number of available ligands is less for ligands randomly placed than for ligands in islands. 
a

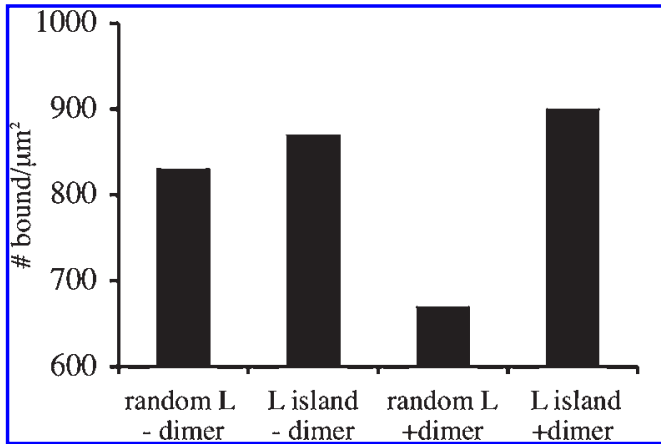

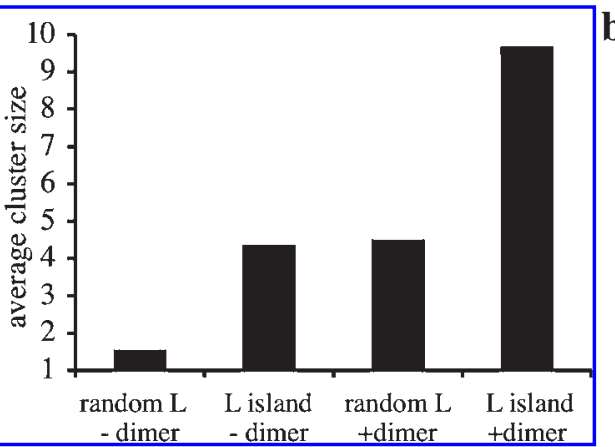

FIG. 6. Effect of dimerization and arranged or randomly placed ligand on the number of integrins bound and average integrin cluster size. (a) The number of bound integrins and (b) average cluster size are plotted for four cases of integrin dimerization and ligand organization. Parameters: $k_{\text {dimer }}=10^{5} \mathrm{~s}^{-1}, k_{\mathrm{mono}}=10^{3} \mathrm{~s}^{-1}$ for integrins able to dimerize; otherwise $k_{\mathrm{dimer}}$ and $k_{\mathrm{mono}}=$ $0, k_{\text {bind }}=10^{-7} \mathrm{~cm}^{2} / \mathrm{s}, k_{\text {unbind }}=1 \mathrm{~s}^{-1}, k_{\text {move }}=10^{3} \mathrm{~s}^{-1},[\mathrm{~L}]=2400 / \mu \mathrm{m}^{2}$, nine ligands per island, and $15 \mathrm{~nm}$ between ligands in an island.

onstrated in Fig. 7 (left). This competition occurs over the entire range of physiologically relevant conditions: as the amount of integrin clustering increases (by increasing $\left.k_{\text {dimer }}\right)$, the amount of ligand binding decreases. Thus dimerization of integrins, although effective in causing integrin clustering, is not able to increase ligand binding.

\section{Integrin dimerization with binding to ligand islands: both clustering and binding may be increased under appropriate conditions}

We have shown above that, as compared with the case of nondimerizing integrins binding to randomly arranged ligand, (1) allowing integrin dimerization increases cluster size and decreases ligand binding (Fig. 6) and (2) or- ganizing the ligand into ligand islands increases cluster size without affecting ligand binding (Fig. 5b). One might conclude, then, that simulations with both integrin dimerization and ligand islands would further increase the cluster size while decreasing ligand binding. This is indeed true for some parameter ranges. However, with appropriate organization of ligand islands one can also achieve an increase in both the number of bound integrins and the average cluster size (Fig. 6). It is particularly interesting to compare the increase in the number of bound integrins and the average cluster size between randomly placed ligand and ligand islands for integrins able to dimerize. These conditions have significance for the design of experiments, because integrin dimerization is believed to occur in vivo and the placement of ligands is a

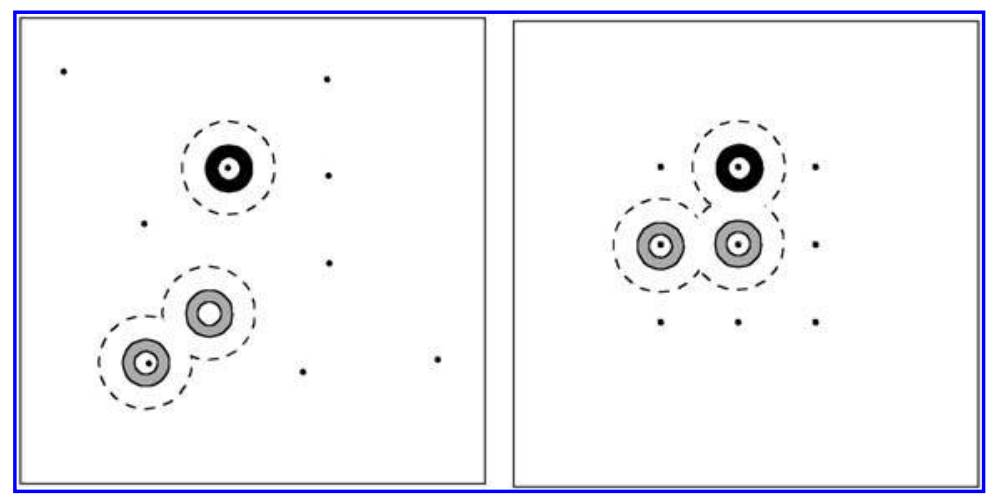

FIG. 7. Dimerizing integrins compete or cooperate to bind randomly placed or organized ligand islands. Integrins are represented as hollow circles and the open central region of the integrin is the region of the integrin able to bind ligand. Dimer pairs of integrins are gray and monomers are black. Ligand peptides are black dots. Integrin clusters are represented with dotted lines. Left: Integrin dimerization with randomly placed ligand reduces the number of integrins bound to ligand. An integrin bound to ligand may remain a monomer because the other integrins are also ligand bound and remain far away. Integrins in a cluster are unable to find free ligand and integrins bound to ligand are too far apart to be in a cluster. Right: Integrin dimerization with ligand arranged in islands can enhance both integrin clustering and ligand binding. When integrin clusters are near a ligand island the local free ligand concentration is high, increasing the chance of an integrin finding a ligand and binding. 
commonly manipulated variable. Our results demonstrate a potential increase in adhesion via both a $35 \%$ increase in the number of bound integrins and an increase in integrin signaling via a 2-fold higher average cluster size, simply due to arranging ligands into islands.

This synergy rather than competition between integrin-integrin and integrin-ligand interactions, in terms of ability to both bind and cluster integrins during an adhesive event, is shown schematically in Fig. 7 (right). Cooperation between ligand binding and integrin clustering is possible when the spacing between islands in an island is similar to that between integrins in a cluster created by dimerization alone.

Our simulation results compare well with experimental data on integrin clustering. Buensuceso et al. ${ }^{16}$ used $\alpha_{\mathrm{IIb}} \beta_{3}$ integrins that had been mutated to allow detection of changes in integrin clustering in live cells. They observed an increase in clustering due to binding a multivalent ligand as compared with monovalent ligand. Our simulations also show an increase in clustering due to binding multivalent ligand (equivalent to a ligand island) compared with monovalent ligand (equivalent to randomly placed ligands) (Fig. 6). In another experiment by the same authors, increased integrin clustering was observed after treatment of the cells with an agent to crosslink integrins. This increase in integrin clustering led to an increase in integrin binding to multivalent ligand, but not monovalent ligand. Our simulations show the same qualitative behavior in response to integrin clustering via dimerization.
The arrangement of ligands into islands could increase the average cluster size by changing the distribution of integrins in multiple ways. Therefore we investigated the effect of the organization of ligands available for binding to dimerization-capable integrins on the cluster size distribution (Fig. 8). With no ligand present, a small amount of partner switching is possible and most integrins are in small clusters. When ligand is arranged randomly, the integrins are able to form larger clusters because binding of immobilized ligand reduces the effective diffusion of integrins, favoring partner switching. Finally, when ligand is arranged into islands, most of the integrins are in large clusters because the organization of ligand islands complements integrin dimerization. These results show that arranging ligand into islands allows large integrin clusters to form, possibly creating nucleation sites for adhesion complex formation.

\section{Size of ligand islands and spacing within islands affect ligand binding and cluster size}

We also examined the effect of island size on integrin binding over a broad range of ligand island sizes. We varied the number of ligands per island from 1 to 100 at a constant concentration of ligand (Fig. 9a). Random organization of ligand (one ligand per island) gives a low number of bound integrins and a small cluster size. A ligand island of nine ligands per island produces the largest number of bound ligands. Similarly, average cluster size peaks at a ligand island size of 16 ligands per island.

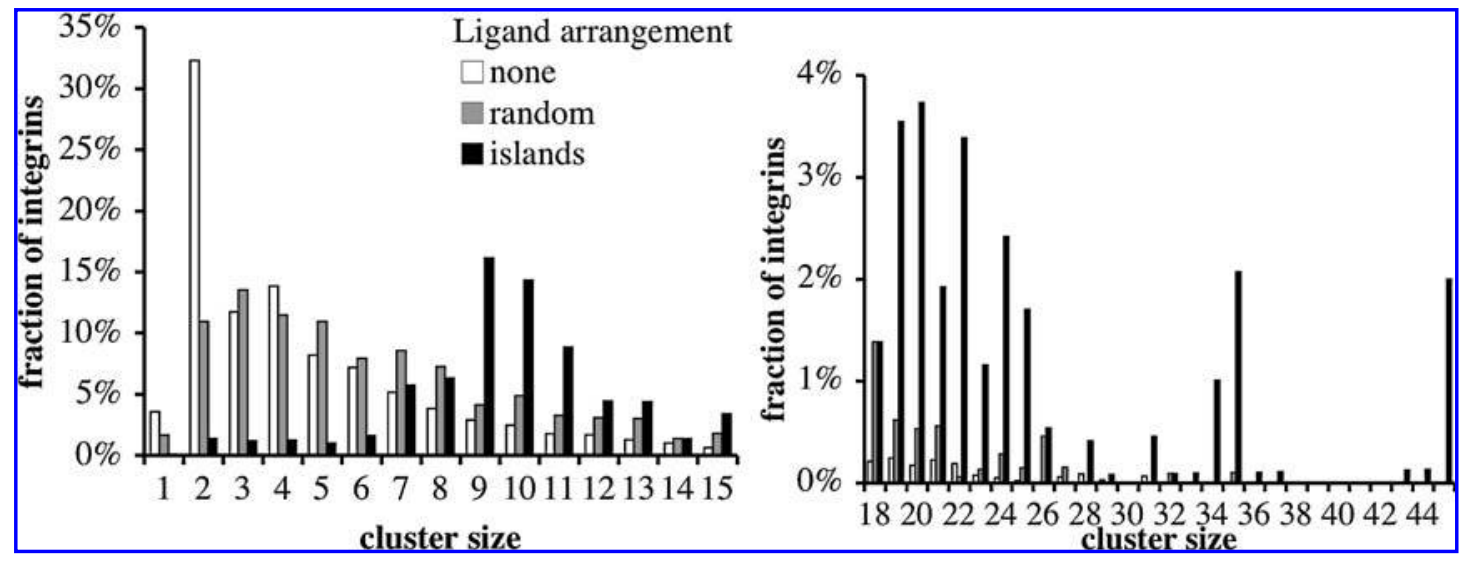

FIG. 8. Cluster size distribution. Shown are representative plots of the distribution of cluster sizes for integrin dimerization: with no ligand, with random ligand, and with ligand islands. With no ligand present, the average cluster size is 3.1 and $15 \%$ of the integrins are in clusters larger than size 8. Partner switching was able to occur and large clusters were formed. When ligand is arranged randomly, the average cluster size is 4.5 and $28 \%$ of integrins are in clusters larger than size 8 . The average cluster size increased because binding of immobilized ligand reduces the effective diffusion of integrins, favoring partner switching. Finally, when ligand is arranged into islands, the average cluster size is increased to 9.7 by the formation of larger clusters, $82 \%$ of integrins are in clusters larger than size 8 . The same conditions were used as in Fig. 6 . The total concentration of ligand was $2400 / \mu \mathrm{m}^{2}$. 


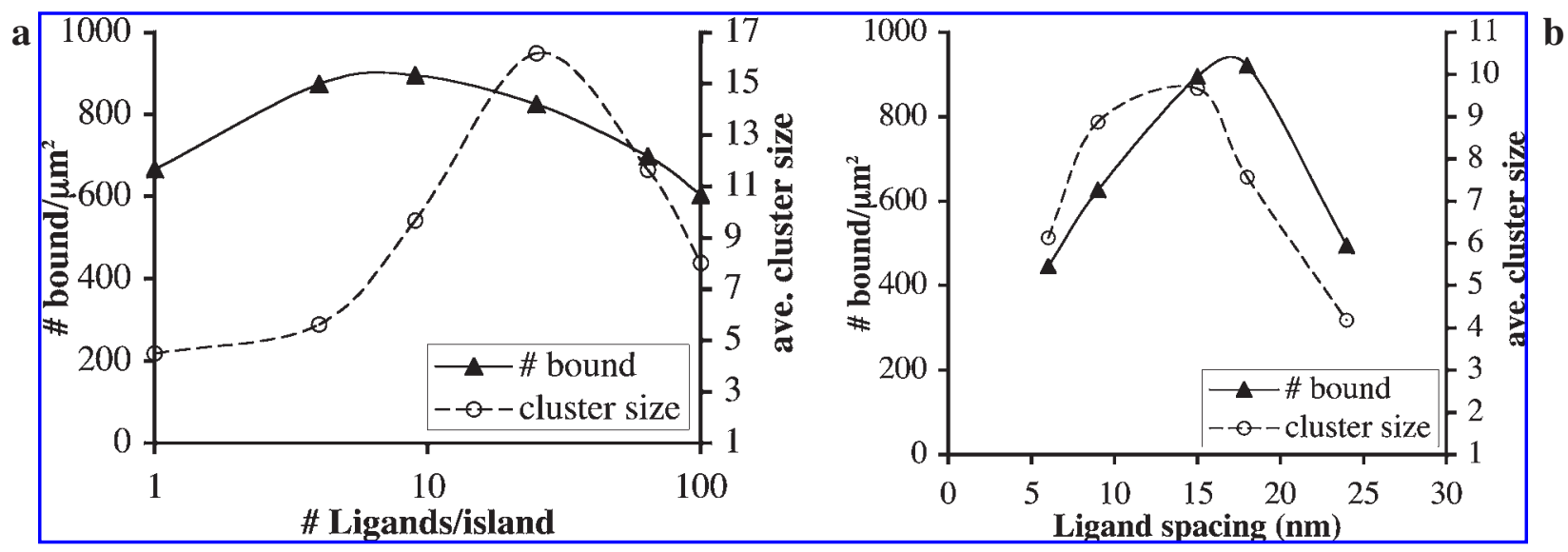

FIG. 9. Effect of island parameters. (a) Effect of island size. The number of bound integrins and average cluster size versus the number of ligands per island is plotted for ligands arranged in islands. The ligand spacing is $15 \mathrm{~nm}$. (b) Effect of the spacing between ligands in an island. The number of bound integrins and average cluster size versus ligand spacing within an island is plotted for ligand arranged in islands (nine ligands per island). Parameters: $k_{\text {dimer }}=10^{5} \mathrm{~s}^{-1}, k_{\text {mono }}=10^{3} \mathrm{~s}^{-1}, k_{\mathrm{bind}}=10^{-7}$ $\mathrm{cm}^{2} / \mathrm{s}, k_{\text {unbind }}=1 \mathrm{~s}^{-1}, k_{\text {move }}=10^{5} \mathrm{~s}^{-1},[\mathrm{~L}]=2400 / \mu \mathrm{m}^{2}$.

These results are consistent with the data of Maheshwari et al. ${ }^{28}$ and Koo et al, ${ }^{9}$ showing an increase in fibroblast migration speed and fibroblast adhesion, respectively, as the ligand island size is increased from one to five or nine. Our simulation results show that island sizes of 9-16 ligands will promote the most integrin clustering and ligand binding and suggest that further increases in island size may produce submaximal adhesion. Islands of 9-16 ligands may be optimal for promoting cell adhesion and perhaps cellular responses dependent on adhesion.

The spacing of ligands within an island has a significant role in the availability of ligand. If two ligands are closer together than the space between the binding sites of two adjacent integrins $(9 \mathrm{~nm})$, the ligands will not both be available to bind integrin. If the ligands in an island are further apart than the maximum distance at which two integrins can remain dimers $(21 \mathrm{~nm})$, the integrins will not be able to bind both ligands and dimerize. Our simulations show that ligand binding and integrin clustering are maximized when the ligand spacing is intermediate between the two extremes of 9 and $21 \mathrm{~nm}$, approximately 14-18 nm (Fig. 9b). Our results also suggest that ligand spacing outside of this range of 14-18 $\mathrm{nm}$ results in a drastically reduced amount of ligand binding and integrin clustering, suggesting reduced cell adhesion.

\section{DISCUSSION}

One major result of this work is the demonstration that integrin dimerization can drive the oligomerization of in- tegrins, that is, the generation of integrin clusters containing more than two integrins. We show that partner switching, the rapid switching of dimerization bonds between neighboring integrins, can create large integrin clusters. In agreement with simulations of $\mathrm{G}$ protein-coupled receptor dimerization, ${ }^{18}$ partner switching is most significant when dimerization is weak. The weak dimerization of integrins is supported by the values of $K_{\mathrm{D} \text {, dimer }}^{3 \mathrm{~d}}$ reported in the literature. ${ }^{12}$

Another key finding in this work is the demonstration that integrin dimerization and ligand organization into islands can cooperate to significantly increase both integrin binding and clustering, as shown in Fig. 6. Integrin binding to ligand and integrin clustering are increased because an integrin cluster near a ligand island creates a high local concentration of integrins and ligands. Integrin dimerization or ligand organization separately cannot increase the number of bound integrins significantly.

While it is known that clustering of integrins is necessary to initiate integrin signaling and the formation of adhesion complexes, the specific attributes of an integrin cluster that lead to adhesion complex formation are not clear. A likely requirement is surpassing a threshold number of integrins in a cluster to initiate sufficient signaling to recruit cytoplasmic molecules. As shown in Fig. 8 , when ligands are arranged into islands large clusters are created. In this way, integrin dimerization creates large integrin clusters that may act as nucleation sites for adhesion complex formation. Therefore, adhesion complex formation and cellular adhesion may be facilitated when ligand is organized into islands as compared with when ligand is randomly arranged. 


\section{Avidity modulation}

Two proposed mechanisms for the quantitative modulation of adhesion strength are increasing the affinity between receptor and ligand (termed affinity modulation) and increasing the availability of ligands or receptors. Both mechanisms could enhance the avidity, the overall tendency of a ligand to be bound by receptors at equilibrium. Many forms of avidity modulation may have simultaneous roles in modulating the adhesion strength of cells. Evaluating the relative influence of each of these different mechanisms will have implications for therapeutic strategies, for example, in cancer cell metastasis and immune system disorders or in the design of biomimetic surfaces for use in tissue engineering.

Affinity modulation of integrin receptors has been implicated in many cell types, for example, in platelet aggregation. ${ }^{39}$ In a mathematical model of affinity modulation, receptors that became located in clusters were then assigned an enhanced affinity for ligand, effectively increasing the strength per bond. ${ }^{40}$ When clustered ligands were presented to the surface, the number of bound receptors increased as receptors by chance diffused next to a cluster, received that higher affinity, and bound ligand more tightly. This mechanism for affinity modulation appears to be a plausible explanation of the enhanced cellular adhesion in response to clustered ligand for cells that demonstrate affinity modulation.

However, other types of avidity modulation have complementary roles in changing the adhesion strength of cells. The clustering of integrins through chemical crosslinking has been shown to increase ligand binding, even in cells that show significant affinity modulation. ${ }^{4}$ In some cells, affinity modulation does not appear to play a role. ${ }^{41}$ Our simulations demonstrate that integrin dimerization can cause clustering of integrins and, in combination with specific ligand organization, make free integrins more likely to find free ligand. In this way, integrin dimerization can increase the number of bound integrins at equilibrium, increasing the avidity of integrin-ligand binding.

\section{Design of biomimetic surfaces for tissue engineering}

Controlling adhesion and cell function in therapeutic and diagnostic techniques will in part be accomplished by designing new biomimetic surfaces. ${ }^{42}$ Our simulations of integrin dimerization and ligand binding show that the amount of ligand binding and integrin clustering are increased significantly when ligand is organized into islands instead of placed randomly on the surface (Fig. 6). Surfaces with organized ligands have shown the importance of ligand organization on cell adhesion. ${ }^{9,28}$

The design of biomimetic surfaces will need to consider three key variables: the total ligand concentration, the number of ligands per island, and the spacing of ligand within the island. These variables are not independent. The total ligand concentration is proportional to the number of ligands per island and inversely proportional to the spacing between ligands within an island. The impact of total ligand concentration on cell adhesion has previously been investigated for random placement of ligand. ${ }^{43}$ Our results demonstrate that organized ligand can produce more bound integrins and a larger average cluster size than randomly placed ligand, when the number of ligands per island and the spacing of ligand within the island are appropriate. We suggest that surfaces with ligand arrangements similar to those described herenamely ligand islands of 9-16 ligands per island with a ligand spacing of $15 \mathrm{~nm}$-will have a greater effect on cell adhesion than other ligand arrangements.

The number of ligands per island has different maxima for ligand binding and integrin clustering (see Fig. 9a). Although it is clear that ligand binding and integrin clustering are necessary for adhesion and integrin signaling, the minimum amounts of each are not known and may vary between cell types. Presumably, an island size that has relatively high levels of ligand binding and integrin clustering would give the most cell adhesion.

Here we have shown how to take advantage of the dimerization of integrins and ligand organization for an increased amount of ligand binding and integrin clustering. Our simulations have taken into account the previously neglected role of integrin dimerization in ligand binding and integrin clustering. We expect a significant increase in cell adhesion could be realized by recognizing the impact of integrin dimerization on integrin clustering when designing adhesive surfaces.

\section{ACKNOWLEDGMENTS}

This work was supported by NIH grant GM 062930 (J.J.L.), U.S. Army Research Laboratory and Research Office grant DAAD19-03-1-0168, and NIH training grant GM 08353 (C.J.B.).

\section{REFERENCES}

1. Miyamoto, S., Akiyama, S.K., and Yamada, K.M. Synergistic roles for receptor occupancy and aggregation in integrin transmembrane function. Science 267, 883, 1995.

2. Yauch, R.L., Felsenfeld, D.P., Kraeft, S.K., Chen, L.B., Sheetz, M.P., and Hemler, M.E. Mutational evidence for control of cell adhesion through integrin diffusion/clustering, independent of ligand binding. J. Exp. Med. 186, 1347, 1997.

3. Laplantine, E., Maurer, P., Vallar, L., Eble, J., Paulsson, M., Bruckner, P., Kieffer, N., and Aumailley, M. The integrin $\beta_{1}$ subunit cytoplasmic tail forms oligomers: A po- 
tential role in $\beta_{1}$ integrin clustering. Biol. Cell 94, 375, 2002.

4. Hato, T., Pampori, N., and Shattil, S.J. Complementary roles for receptor clustering and conformational change in the adhesive and signaling functions of integrin $\alpha_{\mathrm{IIb}} \beta_{3}$.. Cell Biol. 141, 1685, 1998.

5. Aukhil, I., Joshi, P., Yan, Y., and Erickson, H.P. Cell- and heparin-binding domains of the hexabrachion arm identified by teascin expression proteins. J. Biol. Chem. 268, 2542, 1993.

6. Hynes, R.O., and Yamada, K.M. Fibronectins: Multifunctional modular glycoproteins. J. Cell Biol. 95, 369, 1982.

7. Alsberg, E., Anderson, K.W., Albeiruti, A., Franceschi, R.T., and Mooney, D.J. Cell-interactive alginate hydrogels for bone tissue engineering. J. Dent. Res. 80, 2025, 2001.

8. Rowley, J.A., Sun, Z.X., Goldman, D., and Mooney, D.J. Biomaterials to spatially regulate cell fate. Adv. Mater. 14, 886, 2002.

9. Koo, L.Y., Irvine, D.J., Mayes, A.M., Lauffenburger, D.A., and Griffith, L.G. Co-regulation of cell adhesion by nanoscale RGD organization and mechanical stimulus. J. Cell Sci. 115, 1423, 2002.

10. Myou, S., Zhu, X., Boetticher, E., Qin, Y., Myo, S., Meliton, A., Lambertino, A., Munoz, N.M., Hamann, K.J., and Leff, A.R. Regulation of adhesion of AML14.3D10 cells by surface clustering of $\beta_{2}$-integrin caused by ERK-independent activation of cPLA2. Immunology 107, 77, 2002.

11. Li, R.H., Babu, C.R., Lear, J.D., Wand, A.J., Bennett, J.S., and DeGrado, W.F. Oligomerization of the integrin $\alpha_{\mathrm{IIb}} \beta_{3}$ : Roles of the transmembrane and cytoplasmic domains. Proc. Natl. Acad. Sci. U.S.A. 98, 12462, 2001.

12. Li, R.H., Mitra, N., Gratkowski, H., Vilaire, G., Litvinov, R., Nagasami, C., Weisel, J.W., Lear, J.D., DeGrado, W.F., and Bennett, J.S. Activation of integrin $\alpha_{\mathrm{IIb}} \beta_{3}$ by modulation of transmembrane helix associations. Science 300, 795, 2003.

13. Laukaitis, C.M., Webb, D.J., Donais, K., and Horwitz, A.F. Differential dynamics of $\alpha_{\mathrm{v}}$ integrin, paxillin, and $\alpha$-actinin during formation and disassembly of adhesions in migrating cells. J. Cell Biol. 153, 1427, 2001.

14. Miranti, C.K., Leng, L., Maschberger, P., Brugge, J.S., and Shattil, S.J. Identification of a novel integrin signaling pathway involving the kinase Syk and the guanine nucleotide exchange factor Vav1. Curr. Biol. 8, 1289, 1998.

15. Obergfell, A., Eto, K., Mocsai, A., Buensuceso, C., Moores, S.L., Brugge, J.S., Lowell, C.A., and Shattil, S.J. Coordinate interactions of Csk, Src, and Syk kinases with $\alpha_{\mathrm{IIb}} \beta_{3}$ initiate integrin signaling to the cytoskeleton. J. Cell Biol. 157, 265, 2002.

16. Buensuceso, C., de Virgilio, M., and Shattil, S.J. Detection of integrin $\alpha_{\mathrm{IIb}} \beta_{3}$ clustering in living cells. J. Biol. Chem. 278, 15217, 2003.

17. Wiseman, P.W., Brown, C.M., Webb, D.J., Hebert, B., Johnson, N.L, Squier, J.A., Ellisman, M.H., and Horwitz, A.F. Spatial mapping of integrin interactions and dynamics during cell migration by image correlation microscopy. J. Cell Sci. 117, 5521, 2004.

18. Woolf, P.J., and Linderman, J.J. Self organization of membrane proteins via dimerization. Biophys. Chem. 104, 217, 2003.
19. Lauffenburger, D., and Linderman, J.J. Receptors: Models for Binding, Trafficking, and Signaling. New York: Oxford University Press, 1993.

20. Petit, V., and Thiery, J.P. Focal adhesions: Structure and dynamics. Biol. Cell 92, 477, 2000.

21. Chothia, C., and Jones, E.Y. The molecular structure of cell adhesion molecules. Annu. Rev. Biochem. 66, 823, 1997.

22. Labrador, V., Riha, P., Muller, S., Dumas, D., Wang, X., and Stoltz, J.F. The strength of integrin binding between neutrophils and endothelial cells. Eur. Biophys. J. 32, 684, 2003.

23. Walsh, M.T., Ryan, M., Hillmann, A., Condren, R., Kenny, D., Dinan, T., and Thakore, J.H. Elevated expression of integrin $\alpha_{\mathrm{II}} \beta_{3}$ in drug-naive, first-episode schizophrenic patients. Biol. Psychiat. 52, 874, 2002.

24. Akiyama, S.K., and Yamada, K.M. The interaction of plasma fibronectin with fibroblastic cells in suspension. $\mathbf{J}$. Biol. Chem. 260, 4492, 1985.

25. Faull, R.J., Kovach, N.L., Harlan, J.M., and Ginsberg, M.H. Affinity modulation of integrin $\alpha_{\mathrm{v}} \beta_{1}$ : Regulation of the functional response by soluble fibronectin. J. Cell Biol. 121, 155, 1993.

26. Otoole, T.E., Loftus, J.C., Du, X.P., Glass, A.A., Ruggeri, Z.M., Shattil, S.J., Plow, E.F., and Ginsberg, M.H. Affinity modulation of the $\alpha_{\mathrm{IIb}} \beta_{3}$ integrin (platelet GPIIb-Ia) is an intrinsic property of the receptor. Cell Regul. 1, 883, 1990.

27. Thiel, M., Zourelidis, C., Chambers, J.D., von Andrian, U.H., Arfors, K.E., Messmer, K., and Peter, K. Expression of $\beta_{2}$ integrins and L-selectin on polymorphonuclear leukocytes in septic patients. Eur. Surg. Res. 29, 160, 1997.

28. Leahy, D.J., Aukhil, I., and Erickson, H.P. 2.0 angstrom crystal structure of a four-domain segment of human fibronectin encompassing the RGD loop and synergy region. Cell 84, 155, 1996.

29. Maheshwari, G., Brown, G., Lauffenburger, D.A., Wells, A., and Griffith, L.G. Cell adhesion and motility depend on nanoscale RGD clustering. J. Cell Sci. 113, 1677, 2000.

30. Broday, D.M. Diffusion of clusters of transmembrane proteins as a model of focal adhesion remodeling. Bull. Math. Biol. 62, 891, 2000.

31. Rowley, R.L. Statistical Mechanics for Thermophysical Property Calculations. Englewood Cliffs, NJ: PrenticeHall, 1994.

32. Ward, M.D., and Hammer, D.A. Focal contact assembly through cytoskeletal polymerization: Steady-state analysis. J. Math. Biol. 32, 677, 1994.

33. Bell, G.I. Models for specific adhesion of cells to cells. Science 200, 618, 1978.

34. Shoup, D., and Szabo, A. Role of diffusion in ligand binding to macromolecules and cell-bound receptors. Biophys. J. 40, 33, 1982.

35. Gennis, R.B. In: Cantor, C.R., ed. Biomembranes: Molecular Structure and Function. Springer Advanced Texts in Chemistry. New York: Springer-Verlag, 1989.

36. Lee, I., and Marchant, R.E. Force measurements on the molecular interactions between ligand (RGD) and human platelet $\alpha_{\mathrm{IIb}} \beta_{3}$ receptor system. Surf. Sci. 491, 433, 2001.

37. Goldsmith, H.L., McIntosh, F.A., Shahin, J., and Frojmovic, M.M. Time and force dependence of the rupture 
of glycoprotein IIb-3a-fibrinogen bonds between latex spheres. Biophys. J. 78, 1195, 2000.

38. Pierschbacher, M.D., and Ruoslahti, E. Cell attachment activity of fibronectin can be duplicated by small synthetic fragments of the molecule. Nature 309, 30, 1984.

39. Shattil, S.J., Kashiwagi, H., and Pampori, N. Integrin signaling: The platelet paradigm. Blood 91, 2645, 1998.

40. Irvine, D.J., Hue, K.A., Mayes, A.M., and Griffith, L.G. Simulations of cell-surface integrin binding to nanoscaleclustered adhesion ligands. Biophys. J. 82, 120, 2002.

41. van Kooyk, Y., and Figdor, C.G. Avidity regulation of integrins: The driving force in leukocyte adhesion. $\underline{\text { Curr. }}$ Opin. Cell Biol. 12, 542, 2000.

42. Griffith, L.G. Emerging design principles in biomaterials and scaffolds for tissue engineering. Ann. N.Y. Acad. Sci. 961, 83, 2002.
43. Massia, S.P., and Hubbell, J.A. An RGD spacing of 440 $\mathrm{nm}$ is sufficient for integrin $\alpha_{\mathrm{v}} \beta_{3}$-mediated fibroblast spreading and $140 \mathrm{~nm}$ for focal contact and stress fiber formation. J. Cell Biol. 114, 1089, 1991.

Address reprint requests to: Jennifer J. Linderman, Ph.D. Department of Chemical Engineering 3074 H.H. Dow Building, 2300 Hayward Street University of Michigan Ann Arbor, MI 48109-2136

E-mail: linderma@umich.edu 
This article has been cited by:

1. T. Yang, M. H. Zaman. 2009. Regulation of Cell Adhesion Free Energy by External Sliding Forces. Experimental Mechanics 49:1, 57-63. [CrossRef]

2. Tanyarut Boontheekul, Hyun-Joon Kong, Susan X. Hsiong, Yen-Chen Huang, L. Mahadevan, Herman Vandenburgh, David J. Mooney. 2008. Quantifying the relation between bond number and myoblast proliferation. Faraday Discussions 139, 53. [CrossRef]

3. Alexander G Shard, Paul E Tomlins. 2006. Biocompatibility and the efficacy of medical implants. Regenerative Medicine 1:6, 789-800. [CrossRef] 